\title{
Broncoscopia terapéutica en un paciente pediátrico con casi ahogamiento en fosa séptica
}

\author{
Therapeutic bronchoscopy in a pediatric patient \\ with near drowning in a septic tank
}

\author{
José Luis Patiño-Galeana,, Linda Michelle Silva-Lira, ${ }^{*}$ Noemí Torres-Sánchez,* Carlos Montaño-Martínez*
}

*Hospital Regional de Alta Especialidad de Ixtapaluca, Estado de México.

RESUMEN. Introducción: El ahogamiento y el casi ahogamiento son eventos graves y comunes en pacientes pediátricos que de manera habitual se producen en agua dulce y/o salada. La aspiración de contenido orgánico y/o inorgánico es rara. La broncoscopia terapéutica se utiliza en casos aislados. Caso clínico: Se reporta el caso de un paciente masculino de dos años de edad con casi ahogamiento en fosa séptica, su correlación clínica, radiológica y endoscópica. Conclusión: La broncoscopia terapéutica fue el procedimiento complementario para la resolución del evento de aspiración de material orgánico e inorgánico.

Palabras clave: Ahogamiento, casi ahogamiento, fosa séptica, tomografía, broncoscopia.

\section{INTRODUCCIÓN}

El ahogamiento es el proceso de insuficiencia respiratoria por inmersión o sumersión en líquido, con resultados clasificados como muerte, discapacidad o morbilidad. ${ }^{1}$

El casi ahogamiento se define como el paciente que sobrevive al episodio de ahogamiento durante al menos un período de tiempo. ${ }^{2}$ De acuerdo con la Organización Mundial de la Salud el ahogamiento representa la tercera causa de las lesiones no intencionales a nivel mundial y representa 7\% de las muertes por accidente, con 320,000 defunciones estimadas por año. ${ }^{1}$

\section{Correspondencia:}

\section{Dr. José Luis Patiño-Galeana}

Hospital Regional de Alta Especialidad de Ixtapaluca, Estado de México.

Correo electrónico: pepeazul33@gmail.com

Recibido: 12-VI-2020; aceptado: 11-VIII-2020.

Citar como: Patiño-Galeana JL, Silva-Lira LM, Torres-Sánchez N, MontañoMartínez C. Broncoscopia terapéutica en un paciente pediátrico con casi ahogamiento en fosa séptica. Neumol Cir Torax. 2021; 80 (1): 42-45. https:// dx.doi.org/10.35366/99453
ABSTRACT. Introduction: Drowning and near drowning are serious and common events in pediatric patients that usually occur in fresh and/or salt water. Aspiration of organic and/or inorganic content is rare. Therapeutic bronchoscopy is used in isolated cases. Case report: We report the case of a 2 year old male patient with near drowning in the septic tank, its clinical, radiological and endoscopic correlation. Conclusion: Therapeutic bronchoscopy was the complementary procedure for the resolution of the event of aspiration of organic and inorganic material.

Keywords: Drowning, near drowning, septic tank, tomography, bronchoscopy.

La broncoaspiración de material inorgánico es poco común en los eventos de casi ahogamiento, lo cual puede resultar fatal en la mayoría de los sucesos. La broncoscopia terapéutica es de utilidad para la resolución de estos eventos.

\section{CASO CLÍNICO}

Se reporta el caso de un paciente masculino de dos años, sano, quien se encontraba jugando con sus hermanos cerca de una casa habitación en construcción, presenta caída de manera accidental en fosa séptica, lo cual es presenciado por sus hermanos mayores, quienes avisaron a los padres tres minutos después aproximadamente. Éstos lo encuentran en decúbito prono, inconsciente, cubierto por arena, tierra y material orgánico, realizan estimulación directa sobre el tórax sin poder emitir llanto, observan cavidad oral llena de arena y material orgánico por lo que acuden al Hospital Materno Infantil de Ayotzingo sin signos vitales e inconsciente, el paciente presenta paro cardiorrespiratorio que se revierte con un ciclo de reanimación avanzada. Se realiza laringoscopia directa que muestra abundante tierra y material inorgánico en la vía aérea, ésta se asegura y se decide su traslado al Hospital 
Regional de Alta Especialidad de Ixtapaluca, Estado de México, para su tratamiento.

A su ingreso se observa a paciente inconsciente, bajo ventilación con bolsa mascarilla reservorio, presenta compromiso hemodinámico que requiere ingreso a la Unidad de Terapia Intensiva Pediátrica (UTIP) para apoyo de vasopresores e inotrópicos y ventilación mecánica convencional.

El paciente presentó síndrome de distrés respiratorio agudo pediátrico severo (PARDS) que requirió ventilación convencional durante 20 días, el destete de oxígeno se realizó hasta alcanzar saturaciones normales, fue tratado con doble esquema de antibióticos, esteroide sistémico, micronebulizaciones con esteroide y broncodilatador. Se realizaron tres videobroncoscopias flexibles terapéuticas: la primera se efectuó a su ingreso y se observó material inorgánico sólido abundante en la vía aérea, se realizó aspiración y remoción del material referido; en la segunda se observaron datos de traqueoendobronquitis severa, supuración broncopulmonar y zona de isquemia subglótico traqueal, se aspiró material inorgánico sólido; en la tercera broncoscopia se logró la extracción del resto de material sólido (Figura 1).
Los controles radiográficos durante el internamiento mostraron mejoría importante en las zonas de ocupación alveolar, se observaron únicamente algunas bandas de atelectasia. El paciente egresó ventilatoria y neurológicamente estable sin secuelas.

\section{DISCUSIÓN}

El 36\% de los pacientes con casi ahogamiento ocurre en menores de cinco años, ${ }^{3}$ el porcentaje es tres veces más alto en lugares rurales que urbanos, esto se relaciona con el medio socioeconómico y la pobreza. ${ }^{4}$ En México la tasa de mortalidad ha disminuido de manera considerable en la última década, ${ }^{5}$ el grupo etario más frecuente lo ocupan los pacientes entre uno a cuatro años $(48 \%)$, predominando el género masculino. ${ }^{6}$ La falta de barreras o cercados en lugares expuestos al agua y la falta de supervisión de los padres a los niños son las causas más comunes por las cuales ocurren los eventos de ahogamiento. ${ }^{7}$ En el caso del paciente, la falta de señalamiento de la fosa séptica con la inadecuada supervisión fueron las condicionantes para el accidente.
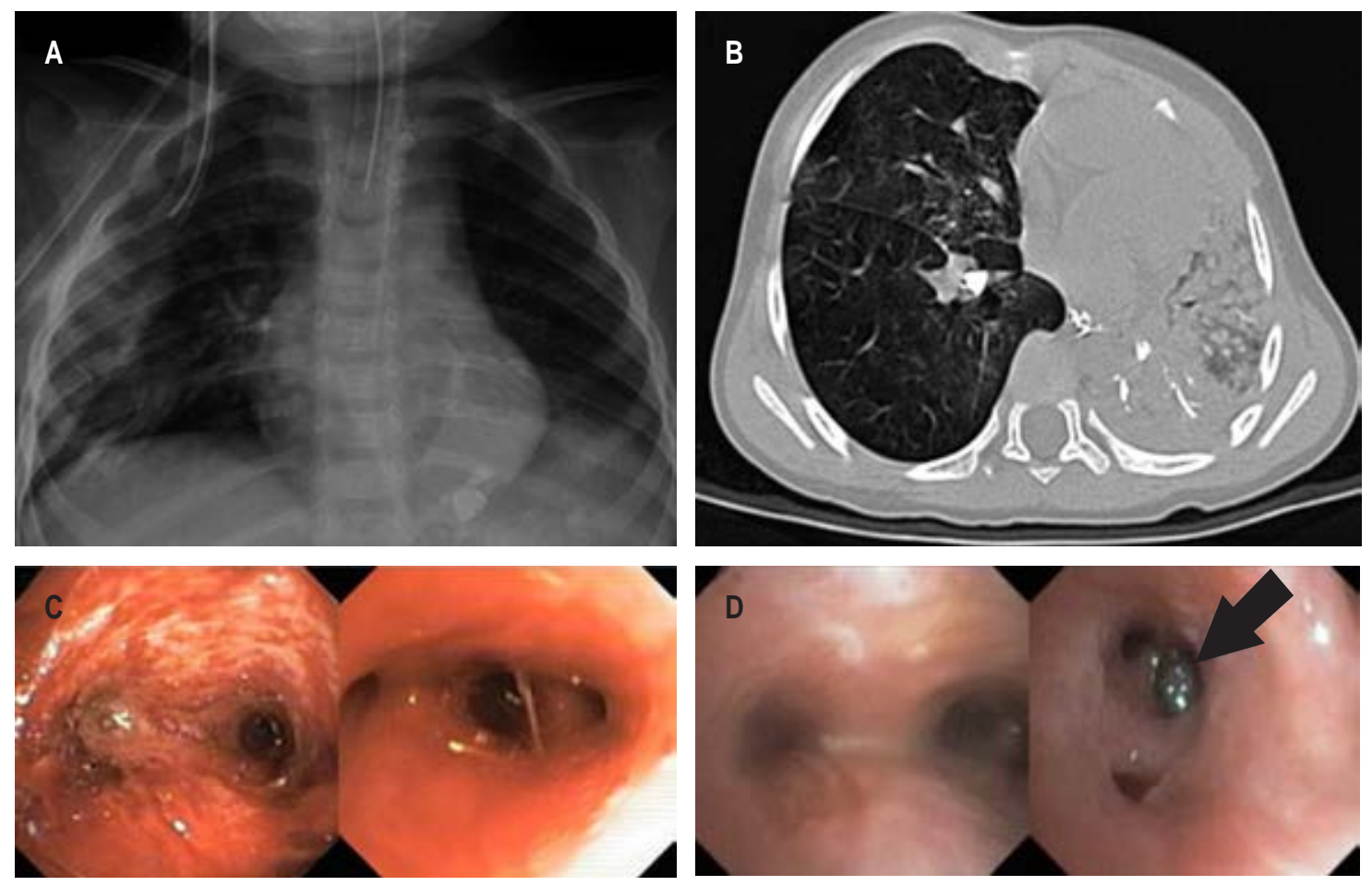

Figura 1: A) Radiografía anteroposterior. Engrosamiento peribronquial de predominio central, ocupación alveolar difusa bilateral, atelectasia basal derecha, estatus de intubación orotraqueal. B) Intubación selectiva del bronquio derecho con colapso pulmonar y elevación secundaria del hemidiafragma en el lado izquierdo. Engrosamiento de las paredes bronquiales, así como imágenes hiperdensas sugestivas de calcificaciones en pared y luz de los trayectos bronquiales segmentarios en forma bilateral y subsegmentarios en el lóbulo inferior izquierdo. C) Broncoscopia flexible. Se observa edema e hiperemia de la mucosa endobronquial moderada con zonas de isquemia sobre la pared anterior de la tráquea precarinal y supuración broncopulmonar bilateral, restos de contenido inorgánico (arena) sobre carina para el lóbulo basal derecho. D) Broncoscopia flexible. Se observa mucosa endobronquial con edema e hiperemia leve con presencia de cuerpo extraño (piedra) que ocluye la entrada del segmento seis derecho. 
El daño secundario a la sumersión es hipoxia, se reporta que menos de $20 \%$ puede presentar laringoespasmo, ${ }^{8}$ el tiempo de resucitación es un factor predictor de buena respuesta cuando se inicia de manera inmediata, lo cual disminuye el período de hipoxia y el daño al parénquima pulmonar, como en el caso del paciente.

Los pacientes que broncoaspiran líquido se asocian con broncoespasmo y atelectasias por inhibición del surfactante con incremento de los cortocircuitos intrapulmonares que producen zonas mal ventiladas. ${ }^{9}$

El paciente presentaba obstrucción de la vía aérea por material orgánico e inorgánico, lo cual condicionó insuficiencia respiratoria y deterioro neurológico, que requirió maniobras avanzadas de reanimación. Se propone a la hipoxia como el principal valor predictor; ${ }^{10}$ sin embargo, existe un puntaje de casi ahogamiento pediátrico que valora el riesgo de lesión posterior a la sumersión con un puntaje del cero al cinco que incluye estado mental normal al momento del ingreso, ausencia de disnea, frecuencia respiratoria normal, ausencia o necesidad de soporte ventilatorio y ausencia de hipotensión (sensibilidad del 82\% y especificidad del 62\%), ${ }_{11}^{11}$ cabe señalar que el paciente presentaba a su ingreso un puntaje de cero. El principal factor predictor de mortalidad es el tiempo de inmersión/ sumersión $(<5 \mathrm{~min})$ y el tiempo inicial de la adecuada resucitación. La anoxia ocurre entre uno a tres minutos. ${ }^{8}$ El porcentaje de supervivencia del paciente en el ingreso era de $12 \%{ }^{9}$

En la radiografía de tórax se observaron zonas radiopacas bilaterales difusas que correspondían a edema alveolar, lo cual a las 24 horas puede ser un predictor clínico posterior al evento de inmersión. ${ }^{12}$ Los cambios radiológicos más comunes corresponden en distrés respiratorio a zonas de ocupación alveolar y atelectasias bilaterales con sensibilidad del $77 \%$ y especificidad del $69 \%$ a las ocho horas del evento. ${ }^{12}$ El tratamiento antibiótico se establece de manera empírica cuando existe la sospecha de aspiración de agua contaminada. ${ }^{13}$ La tomografía axial computarizada proporciona mayor sensibilidad y especificidad para visualizar el parénquima pulmonar y la vía aérea al tratarse de aspiración de material inorgánico (tierra y piedras). En la sospecha fundada de aspiración de cuerpos extraños se encuentra indicada la instrumentación armada de la vía aérea con broncoscopia flexible o rígida con fines diagnósticos y terapéuticos. ${ }^{14}$ En la literatura se reporta el caso de casi ahogamiento por aspiración de tierra en una paciente preescolar con realización de broncoscopia seriada con lavado broncoalveolar con mejoría clínica y radiológica. ${ }^{15}$

\section{CONCLUSIÓN}

La mortalidad en accidentes de ahogamiento en pediatría a nivel mundial es alta. La broncoscopia terapéutica fue el procedimiento complementario para la resolución del evento de aspiración de material orgánico e inorgánico en nuestro paciente. Fue un procedimiento seguro, ya que no se reportaron incidentes ni accidentes.

\section{Agradecimientos}

Agradecemos al personal Regional de Alta Especialidad por su entrega y profesionalismo.

\section{REFERENCIAS}

1. Meddings D, Altieri E, Bierens J, Cassell E, Gissing A, Guevarra J. Preventing drowning: an implementation guide [Internet]. World Health Organization. 2017. 116 p. Available from: http://apps.who.int/iris/bits tream/10665/255196/1/9789241511933-eng.pdf?ua=1

2. Fawcett K, Gerber N, lyer S, De Angulo G, Pusic M, Mojica M. Common conditions requiring emergency life support. Pediatr Rev. 2019;40(5):291-301. https://doi.org/10.1542/pir.2017-0331

3. Lukaszyk C, Mittal S, Gupta M, Das R, Ivers R, Jagnoor J. The impact and understanding of childhood drowning by a community in West Bengal, India, and the suggested preventive measures. Acta Paediatr. 2019;108(4):731-739. https://doi.org/10.1111/apa.14592

4. Liu Z, Kong F, Yin L, Wang A, Xiong L, Xie D, et al. Epidemiological characteristics and influencing factors of fatal drowning in children under 5 years old in Hunan Province, China: case-control study. BMC Public Health. 2019;19(1):955. https://doi.org/10.1186/s12889019-7241-z

5. Lin CY, Wang LY, Lu TH. Changes in drowning mortality rates and quality of reporting from 2004-2005 to 2014-2015: A comparative study of 61 countries. BMC Public Health. 2019;19(1):1391. https:// doi.org/10.1186/s12889-019-7749-2

6. Cantón SF, Martínez AMH, Uribe RV. Mortalidad por ahogamiento en la población de menores de 15 años en México, período 1998-2010. Bol Med Hosp Infant Mex. 2012;69(4):321-324.

7. Mintegi S, Esparza MJ, González JC, Rubio B, Sánchez F, Vila JJ, et al. Recomendaciones sobre la prevención de intoxicaciones. An Pediatría. 2015;83(6):440.e1-440.e5. https://doi.org/10.1016/j. anpedi.2015.01.003

8. Quan L, Bierens JJ, Lis R, Rowhani-Rahbar A, Morley P, Perkins GD. Predicting outcome of drowning at the scene: A systematic review and meta-analyses. Resuscitation. 2016;104:63-75. doi: 10.1016/j. resuscitation.2016.04.006.

9. Mott TF, Latimer KM. Prevention and treatment of drowning. Am Fam Physician. 2016;93(7):576-582.

10. Cantu RM, Pruitt CM, Samuy N, Wu CL. Predictors of emergency department discharge following pediatric drowning. Am J Emerg Med [Internet]. 2018;36(3):446-449. Available from: https://doi. org/10.1016/j.ajem.2017.08.057

11. Shenoi RP, Allahabadi S, Rubalcava DM, Camp EA. The pediatric submersion score predicts children at low risk for injury following submersions. Acad Emerg Med. 2017;24(12):1491-1500. https://doi. org/10.1111/acem.13278

12. Ho BJ, Crowe JE, Dorfman SR, Camp EA, Yusuf S, Shenoi RP. Correlation of clinical and chest radiograph findings in pediatric submersion cases. Pediatr Radiol. 2020;50(4):492-500. https://doi. org/10.1007/s00247-019-04588-x 
13. Forler J, Carsin A, Arlaud K, Bosdure E, Viard L, Paut O, et al. Complications respiratoires des noyades accidentelles chez l'enfant. Arch Pediatr. 2010;17(1):14-18. https://doi.org/10.1016/j. arcped.2009.09.021

14. Cuevas-Schacht F, Ortega-Iglesias JC, Sosa-de Martínez MC, Garrido-Galindo C, Pérez-Fernández LF. Broncoscopia en pacientes pediátricos con atelectasia persistente. Hallazgos endoscópicos y resultados de la aspiración selectiva (1990-1992). Acta Pediatr Mex. 2005;26(2):626.
15. Kapur N, Slater A, McEniery J, Greer ML, Masters IB, Chang AB. Therapeutic bronchoscopy in a child with sand aspiration and respiratory failure from near drowning--case report and literature review. Pediatr Pulmonol. 2009;44(10):1043-1047. https://doi. org/10.1002/ppul.21088

Conflicto de intereses: Los autores declaran no tener conflicto de intereses. 